\title{
Simulation Based Comparative Analysis of Resonant Frequency for Tunable Split Ring Resonator in Different Configurations
}

\author{
Muhammad Shoaib ${ }^{1}$, Muhammad Naveed ${ }^{1}$, Tahir Ejaz ${ }^{2}$, Tahir Zaidi ${ }^{1}$, M. Haris Amir ${ }^{1}$, Mashhood Ahmad ${ }^{1}$ \\ ${ }^{1}$ Department of Electrical Engineering, College of Electrical \& Mechanical Engineering, \\ National University of Sciences \& Technology (NUST), Islamabad, Pakistan. \\ ${ }^{2}$ Department of Electrical Engineering, \\ HITEC University, Taxila, Pakistan.
}

\begin{abstract}
Split ring resonators (SRR) are being researched for material sensing applications recently. These structures are easily fabricated and offer moderate quality factor $(Q)$ and resonant frequencies $\left(f_{r}\right)$. These structures provide limited tunability of $f_{r}$ although considerable variation in $Q$ has been established. A simulation based analysis has been carried out to find most appropriate and practical configuration of SSRs to provide considerable shift in $f_{r}$. Simulation results show efficacy of vertically stacked SRR vis-à-vis others configurations to achieve tunability.
\end{abstract}

Keywords—split ring resonator, tunability, vertically stacked split rings, complimentary split rings.

\section{Introduction}

SSRs in various configurations have become an important component for design at microwave frequencies. These components provide low phase noise, moderate quality factor and ease of fabrication, even at minute scales. Recent advances in thin films processing have materialized these devices at nanometer levels. As size of split ring (SR) based resonant devices is very small as compared to the incident electromagnetic radiation, their use in development of metamaterials for terahertz frequencies have also been reported [1].

SR based resonator designs are being researched for fluid sensing and compositional analysis [2], [3] including biomedical sensors [4]. These designs primarily employ cavity perturbation methods to sense shift in $f_{r}$ and change in $Q$ when small amount of homogeneous solution of liquids is placed between the capacitive gap/split. These two parameters depend upon the dielectric constant of the resonator [5].

In this paper, a simulation based analysis of various design parameters is presented to find a most suitable and practically viable option for realization of tunable SSR. Tunable SSRs also form one of the basic configurations of unit cells for meta-materials.

\section{Authors Names / Affiliations \\ Muhammad Shoaib \\ National University of Sciences \\ and Technology, Pakistan. \\ Tahir Zaidi \\ National University of Sciences and Technology, Pakistan.}

M. Haris Amir

National University of Sciences and Technology, Pakistan.
Muhammad Naveed

National University of Sciences and Technology, Pakistan.

Tahir Ejaz HITEC University, Pakistan.

Mashhood Ahmad

National University of Sciences and Technology, Pakistan.

\section{Theory}

SSR can be modeled using $L C$ network; inductive component comprises of length of wire which make the ring a single turn inductor. Gap/split faces and surface of the ring contribute to the total capacitance of SR [6]. Magnetic field surrounds the SR loop whereas conduction current and displacement current flows in circumferential direction on surface of loop and gap respectively [7]. The $f_{r}$ of a SSR is given as

$$
\mathrm{f}_{\mathrm{r}}=\frac{1}{2 \pi \sqrt{L C}}
$$

where $L$ is the overall inductance of SR and $C$ is the overall capacitance. Current flows on the inner wall of cavity and inner surface of SR due to skin depth [8] of material. A simple SSR is shown in Figure 1. A detailed simulation based comparative analysis of various SSR configurations has been evaluated [9]; inferring that each configuration needs its own set of mathematical equations to calculate the $f_{r}$. $f_{r}$ of a cylindrical cavity can be easily controlled by the perturbation method mainly by area perturbation or cavity perturbation [10], [11].

SSRs can be divided into two broad categories; resonators employing single SR and resonators employing multiple SRs. This paper focuses on configurations based on single and two circular split rings placed inside a cylindrical cavity.

For a single SR based resonator, $f_{r}$ predominantly depends upon the radius of the SR [12]. Design equation were developed by Rowe, David J., et al. for $f_{r}$ and $Q$ as a function of SR radius by curve fitting of simulated data

$$
\begin{gathered}
\mathrm{f}_{r}=0.0168 r^{2}-0.502 r+5.19 \\
Q=-8.8 r^{2}+78 r+1810
\end{gathered}
$$

where $r$ is the radius of split ring.

Another method to control the $f_{r}$ by broadside coupling has been demonstrate [13] with small variation of vertical gap (levitation) between two SR. A large number of configurations of stacked SRs have been simulated and discussed in this paper. These variations have also been discussed from the point of view of ease of fabrication and practical implementation. 
Proc. of the Fifth International Conference on Advances in Computing, Electronics and Communication - ACEC 2017. Copyright (C) Institute of Research Engineers and Doctors. All rights reserved.

ISBN: 978-1-63248-121-4 doi: 10.15224/ 978-1-63248-121-4-06

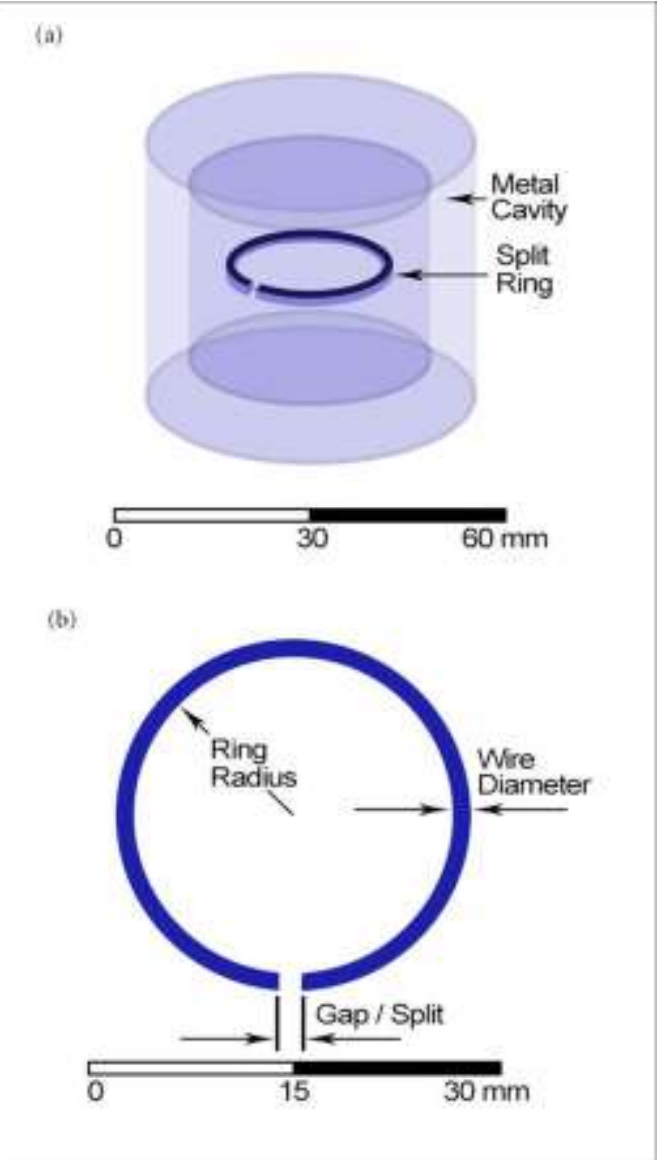

Figure 1 - Design structure for single SSR a) SSR within metallic cylindrical cavity (b) SR showing the gap/ split

\section{Design Structure and Simulation}

ANSYS HFSS ${ }^{\odot}$ (version 13.0) [14] has been used for design of 3-D models and simulation of various configurations of SSRs. These simulations have been grouped in three major categories;

\section{Group-I Cavity with single SSR}

Group-II Cavity with two vertically stacked SSRs (of same dimensions)

Group-III Cavity with complementary SRs (SRs residing within each other)

The dimensions of metallic cylindrical cavity and other optimized parameters [9], [15], [5] used for simulations are shown in Table 1. The material selected for cavity and SRs are Aluminum and Copper respectively [17]. The design structure of single SSR is shown in Figure 1.

Group-I: Cavity with single SSR. Most basic and simple design involves single SR placed inside a metallic cavity as shown in Figure 1(a). This configuration has widely been used for material sensing experiments. Simulation results for $f_{r}$ for

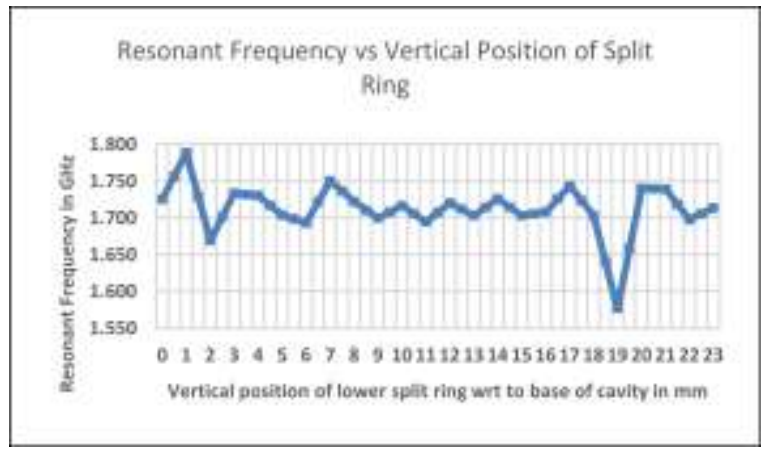

Figure 2a - Resonant frequency of single SSR with variation of vertical position of SR along cavity axis

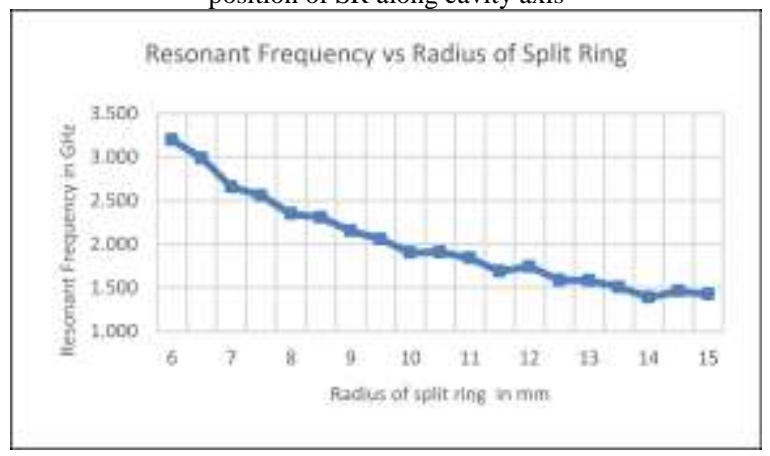

Figure $2 b-$ Shift in resonant Frequency with variation in radius of SR

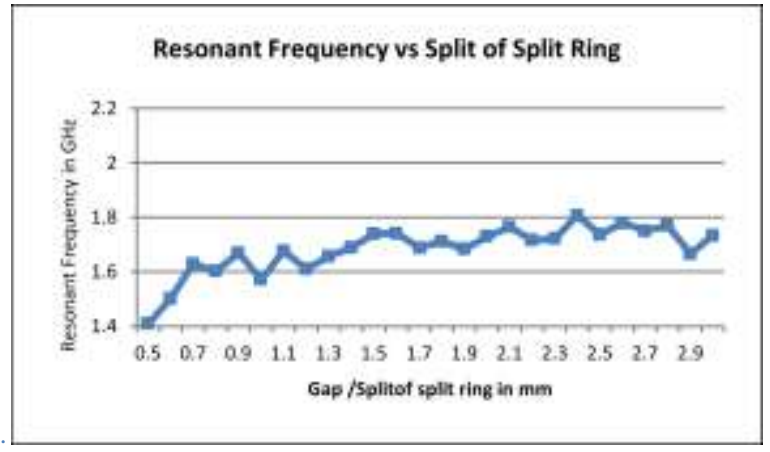

Figure 2c - Shift in resonant Frequency with variation in capacitive gap / split of SR

different vertical positions of SR relative to inner base of cavity are shown in Figure 2a.

\begin{tabular}{lc}
\multicolumn{1}{c}{ TABLE - 1: OPTIMIZED DESIGN PARAMETERS } \\
\hline Pnner diameter of cavity & Design value \\
Shield thickness of cavity & $38 \mathrm{~mm}$ \\
Height of cavity & $7 \mathrm{~mm}$ \\
Capacitive gap (split) or ring & $30 \mathrm{~mm}$ \\
Dimension of ring wire & $1.5 \mathrm{~mm}$ \\
Geometry of ring wire & $1 \mathrm{mmx} 1 \mathrm{~mm}$ \\
Material of cavity & Square \\
Material of rings & Aluminum \\
Space inside cavity & Copper \\
\hline
\end{tabular}

Simulated results confirm shift in $f_{r}$ with change in SR radius [12], as dependence of $f_{r}$ on radius has been linked by Equations (2) and (3). It provides optimum radii of the SR to achieve maximum shift in $f_{r} .6-9 \mathrm{~mm}$ radii exhibit maximum 
shift. The $f_{r}$ for various values of SR radii are plotted in Figure $2 b$.

Surface and gap capacitance of gap / split can also result in shift in $f_{r}$. Figure $2 \mathrm{c}$ shows the $f_{r}$ response for gradual variation of capacitive gap from $0.5 \mathrm{~mm}$ to $3 \mathrm{~mm}$.

Group - II: Cavity with two vertically stacked SSRs. This configuration comprises of two vertically stacked split rings (VS-SR) with gap between them. Lower SR is fixed (static) whereas upper ring is moved upward along the cavity axis thus increasing the gap between two rings. A pneumatic or air assisted mechanism has been demonstrated to achieve levitation of upper ring up to $0.2 \mathrm{~mm}$ [13]. Splits of both the resonators are $180^{\circ}$ out of phase to achieve maximum coupling. Shift in $f_{r}$ is realized by variation of vertical gap between the lower and upper SR. This configuration of SR is shown in Figure-3c.

The vertical gap between the SR was varied from 1 to $10 \mathrm{~mm}$ gradually for two scenarios; lower ring placed $1 \mathrm{~mm}$ above base of cavity and lower ring placed $10 \mathrm{~mm}$ above base of cavity. Simulated results for $f_{r}$ for both the cases are plotted in Figure $3 \mathrm{a}$ and $\mathrm{b}$ respectively. Former scenario shows $\sim 0.5$ $\mathrm{GHz}$ shift in $f_{r}$ with gradual increase in vertical gap between the SR s.

Group - III: Cavity with Complementary SRs (residing within each other). Complementary SRs (one SR is placed inside another SR in the same plane) is another configuration which can provide significant shift in $f_{r}$; when position of one ring is varied with respect to other. Structural dimensions of

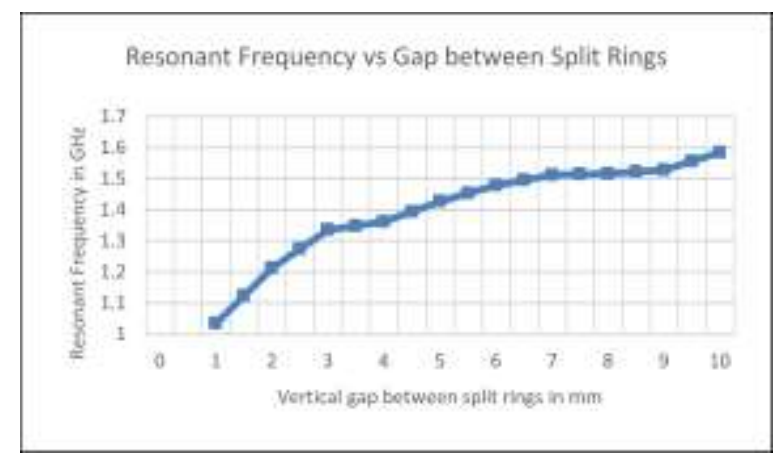

Figure 3a. Lower SR placed $10 \mathrm{~mm}$ above the base of cavity (one third of cavity height) and gap varied from $1 \mathrm{~mm}$ to $10 \mathrm{~mm}$

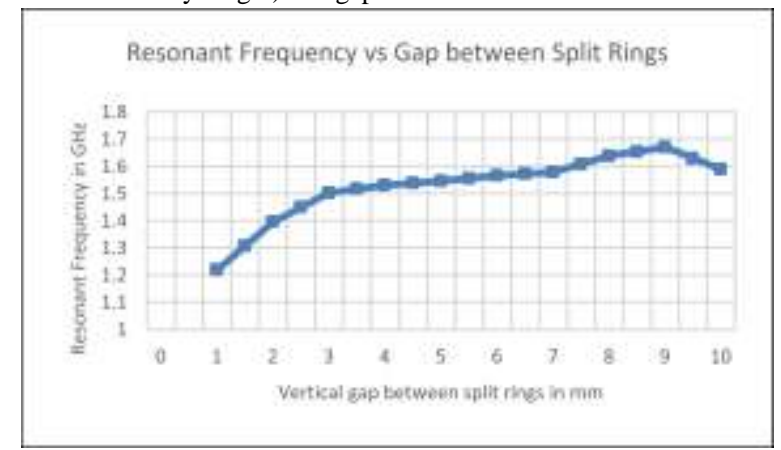

Figure 3b. Lower SR placed $1 \mathrm{~mm}$ above the base of cavity and gap varied from $1 \mathrm{~mm}$ to $10 \mathrm{~mm}$ complementary SRs for this configuration are listed in Table 2 and shown in Figure 3d. The radii of SRs are selected to be between $6-9 \mathrm{~mm}$ as this range of radii has shown maximum shift in $f_{r}$ during simulation of Group-I configurations. Four design variations were simulated for complementary SRs to investigate shift in $f_{r}$.

TABLE - 2: DIMENSION OF COMPLEMENTARY SRS

\begin{tabular}{lc}
\hline \multicolumn{1}{c}{ Parameter } & Design value \\
\hline Radius of outer SR & $8 \mathrm{~mm}$ \\
Radius of outer SR & $6 \mathrm{~mm}$ \\
Capacitive gap (split) or ring & $1.5 \mathrm{~mm}$ \\
Dimension of ring wire & $1 \mathrm{mmx} 1 \mathrm{~mm}$ \\
Geometry of ring wire & Square \\
Material of cavity & Aluminum \\
Material of rings & Copper \\
Space inside cavity & Air \\
\hline
\end{tabular}

Option-1: Inner SR is placed fixed $1 \mathrm{~mm}$ above base of cavity and outer ring is gradually moved upward. The dimensions of ring wire are same for the inner and outer SRs.

Option-2: Outer SR is placed fixed $1 \mathrm{~mm}$ above base of cavity and inner ring is gradually moved upward.

Option-3: Height of inner SR is increased to $10 \mathrm{~mm}$ forming a split cylinder. Split cylinder is placed $1 \mathrm{~mm}$ above base of cavity. Outer SR dimensions are maintained as per Table 2 and gradually moved upward.

Option-4: In this case height of outer SR is increased to $10 \mathrm{~mm}$. Both the rings are placed $1 \mathrm{~mm}$ above base of cavity as initial position. Dimensions of inner SR are kept as per

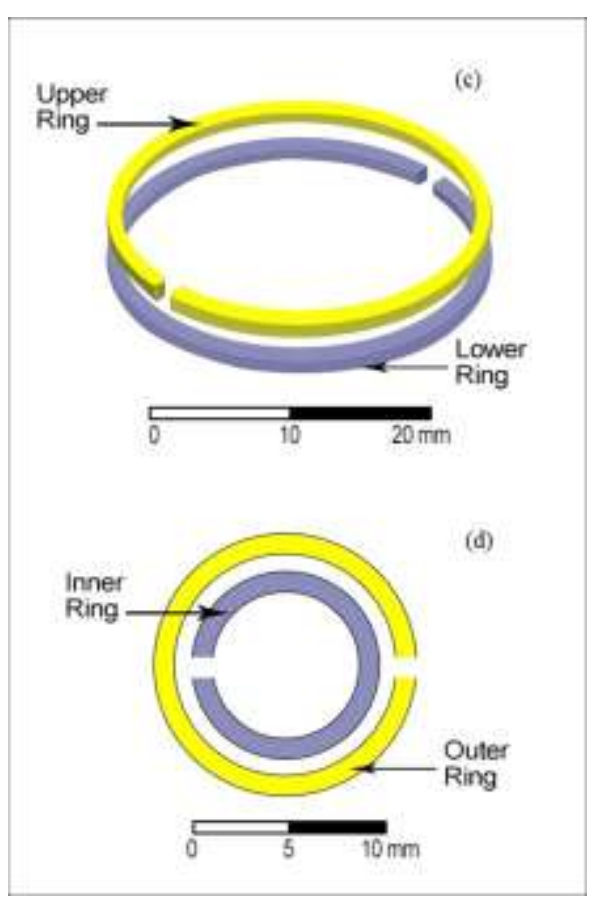

Figure 3 (c) HFSS 3-D model of VS-SR for Group-II configuration (d) complementary SSRs for Group-III configuration 


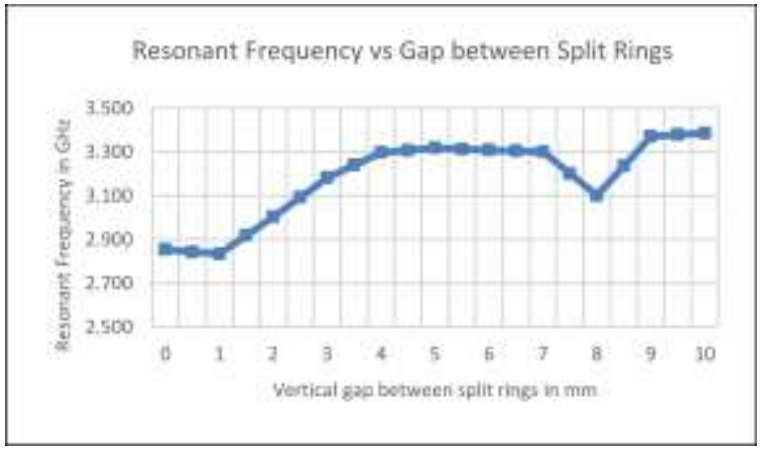

Figure $4 \mathrm{a}$ - Inner ring is moved upward in the cavity whereas outer ring is placed at $1 \mathrm{~mm}$ above base of cavity.

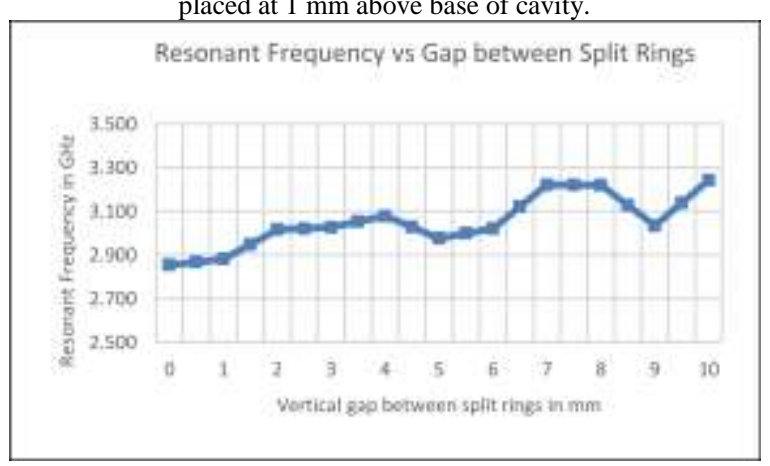

Figure $4 \mathrm{~b}$ - Outer ring is moved upward in the cavity whereas inner ring is placed at $1 \mathrm{~mm}$ above base of cavity.

Table 2. Inner SR is gradually moved upward.

Simulated results of these design variations are plotted in Figures 4a-c.

\section{Analysis and Discussion}

Data of results of three configurations of SRs have been analyzed and discussed with a focus on extent of shift in $f_{r}$ achieved.

Group-I. Change in vertical position of SR inside cavity is the most simple and easy to implement. However, tunability over a considerable broad range could not be achieved. Inner surface areas of cavity and SR remained unchanged in this configuration which can be attributed to a mostly stable $f_{r}$ response.

A huge shift in frequency was observed when radius of SR was varied. Change in radius changed the surface area of SR. This option provides maximum shift in $f_{r}$ for tunability of resonator but it is not practical to change the radius of metallic ring within the cavity. However, this analysis provides an insight to the range of radii of SR which provide maximum shift in $f_{r}$.

Change in gap /split of ring has also been investigated. Very small shift in $f_{r}$ was observed. This can be attributed to presence of surface capacitance of ring which does not change significantly. Overall capacitance of SR is combination of surface capacitance and gap capacitance. Gap capacitance dominates at narrow gaps only [6].

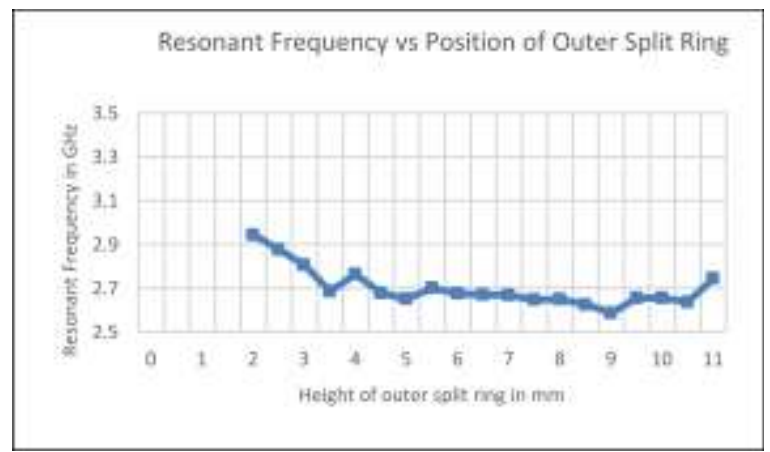

Figure $4 \mathrm{c}$ - Height of inner SR is fixed at $10 \mathrm{~mm}$ and outer split ring is moved up from lower position.

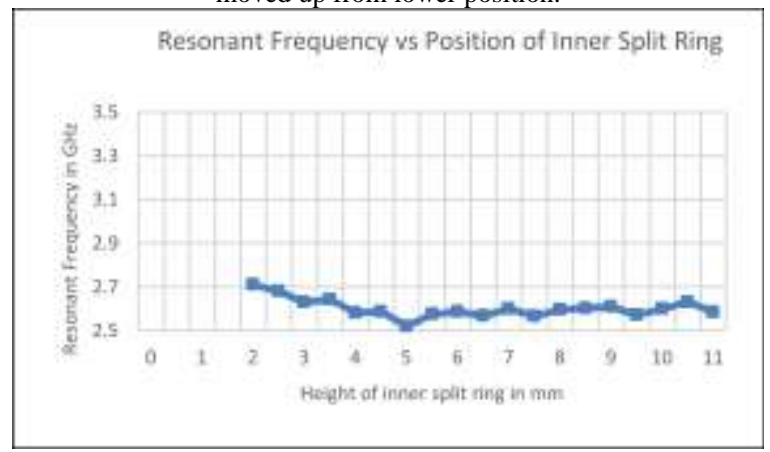

Figure $4 \mathrm{~d}$ - Height of outer split ring is fixed at $10 \mathrm{~mm}$ and inner split ring is moved up from lower position.

Group-II. In case of VS-SRs configuration, considerable shift in $f_{r}$ is achieved in both the simulated configurations. This shift in $f_{r}$ can be attributed to change in the broad side coupling of two SRs as gap between SRs is varied. Increase in vertical gap between the rings reduces the broad side coupling and hence results in shift in $f_{r}$. SRs placed at the bottom of the cavity provide better shift in $f_{r}$.

Both the options in this configuration are easy to manufacture and implement. A mechanism can be introduced to move the upper SR to achieve tunable SSR.

Group-III. Various configurations of complementary SRs have also provided shift in $f_{r}$. Figure 4 shows that this phenomenon is generally less pronounced and maximum shift achieved is also less as compared to Group-II configurations. Furthermore, large variations in adjacent values make it less suitable for tunability. Options 1, 3 and 4 exhibit small shifts in resonant frequency as compared to Option 2.

\section{Comparison of Group I, II and III Configurations.}

Results of HFSS simulations are combined and tabulated in Table 3 and graphically represented in Figure 5. Horizontal axis in Figure 5 represents vertical position of SR and radius of SR in $\mathrm{mm}$ for Group-I ( $2 \mathrm{a}$ and $2 \mathrm{~b}$ ), gap between the SRs in $\mathrm{mm}$ for Group-II ( $3 \mathrm{a}$ and $3 \mathrm{~b}$ ) and vertical position of inner and outer SRs with respect to base of cavity for Group-III (4a to 4d) simulations.

Group II configurations are most suitable and practical to achieve considerable shift in $f_{r}$. These configurations provide $0.549 \mathrm{GHz}$ and $0.451 \mathrm{GHz}$ shift in $f_{r}$ over $10 \mathrm{~mm}$ displacement of upper SR. Practical configurations in Group I 
and Group III provide very less shift in $f_{r}$ as compared to Group II.

Figure 5 sums up the results of all configurations simulated. It is pertinent to notice that for large vertical gap between the SRs for Group II (Group-II inset), the values of $f_{r}$ approach the values for Group I with single SR. This shows that the coupling between the two rings at these values of vertical gap has weakened significantly. Group II configurations show smooth shift as compared to Group III (Group III inset) configurations. This furthers strengthens findings that Group II configurations are best suited toward achieving tunable SSR.

\section{v. Conclusion}

In this paper, a comprehensive simulation based comparative analysis of different configurations of SRs is presented to achieve a considerable shift in resonant frequency $\left(f_{r}\right)$ aimed at realization of a tunable SSR. Three models have been developed, simulated and analyzed. Best practical option to achieve shift in $f_{r}$ with variation of vertical gap between SRs in VS-SRs configuration. Simulations show smooth and considerable shift in $f_{r}$. It is almost impractical to achieve considerable shift in $f_{r}$ with single SSR.

Practical implementation of this configuration for tunable resonators can be used in development of metamaterials. Quality factor $(Q)$ of a resonator is another important performance parameter which needs to be investigated to find a solution with better selectivity of resonators.

TABLE - 3: MINIMUM AND MAXIMUM $f_{r}$ FOR DIFFERENT CONFIGURATIONS OF SSRS

\begin{tabular}{|c|c|c|c|c|c|}
\hline \multirow{2}{*}{\multicolumn{2}{|c|}{ Model }} & \multicolumn{2}{|c|}{$f_{r}(\mathrm{GHz})$} & \multirow{2}{*}{$\begin{array}{l}\text { Max Shift } \\
(\mathrm{GHz})\end{array}$} & \multirow{2}{*}{$\begin{array}{c}\text { Practical } \\
\text { Implementation }\end{array}$} \\
\hline & & Min & Max & & \\
\hline \multirow{2}{*}{ Group-1 } & Change of vertical position of SR from $1 \mathrm{~mm}$ to $25 \mathrm{~mm}$ (Fig 2a) & 1.577 & 1.788 & 0.211 & Yes \\
\hline & Change of radius of SR from $6 \mathrm{~mm}$ to $15 \mathrm{~mm}$ (Fig $2 \mathrm{~b})$ & 1.421 & 3.196 & 1.775 & No \\
\hline \multirow{2}{*}{ Group-2 } & $\begin{array}{l}\text { Lower SR placed } 10 \mathrm{~mm} \text { above the base of cavity and gap varied from } \\
1 \mathrm{~mm} \text { to } 10 \mathrm{~mm} \text { (Fig 3a) }\end{array}$ & 1.585 & 1.035 & 0.549 & Yes \\
\hline & $\begin{array}{l}\text { Lower SR placed } 1 \mathrm{~mm} \text { above the base of cavity and gap varied from } \\
1 \mathrm{~mm} \text { to } 10 \mathrm{~mm} \text { (Fig } 3 \mathrm{~b} \text { ) }\end{array}$ & 1.670 & 1.219 & 0.451 & Yes \\
\hline \multirow{4}{*}{ Group-3 } & $\begin{array}{l}\text { Inner ring is moved upward in the cavity whereas outer ring is placed at } \\
1 \mathrm{~mm} \text { above base of cavity (Fig } 4 \mathrm{a} \text { ) }\end{array}$ & 2.585 & 2.710 & 0.125 & Yes \\
\hline & $\begin{array}{l}\text { Outer ring is moved upward in the cavity whereas inner ring is placed } \\
\text { at } 1 \mathrm{~mm} \text { above base of cavity (Fig } 4 \mathrm{~b} \text { ) }\end{array}$ & 2.853 & 3.242 & 0.389 & Yes \\
\hline & $\begin{array}{l}\text { Height of inner ring is fixed at } 10 \mathrm{~mm} \text { and outer split ring is moved up } \\
\text { from lower position (Fig } 4 \mathrm{c} \text { ) }\end{array}$ & 2.745 & 2.944 & 0.199 & Yes \\
\hline & $\begin{array}{l}\text { Height of outer ring is fixed at } 10 \mathrm{~mm} \text { and inner split ring is moved up } \\
\text { from lower position (Fig 4d) }\end{array}$ & 2.585 & 2.710 & 0.125 & Yes \\
\hline
\end{tabular}

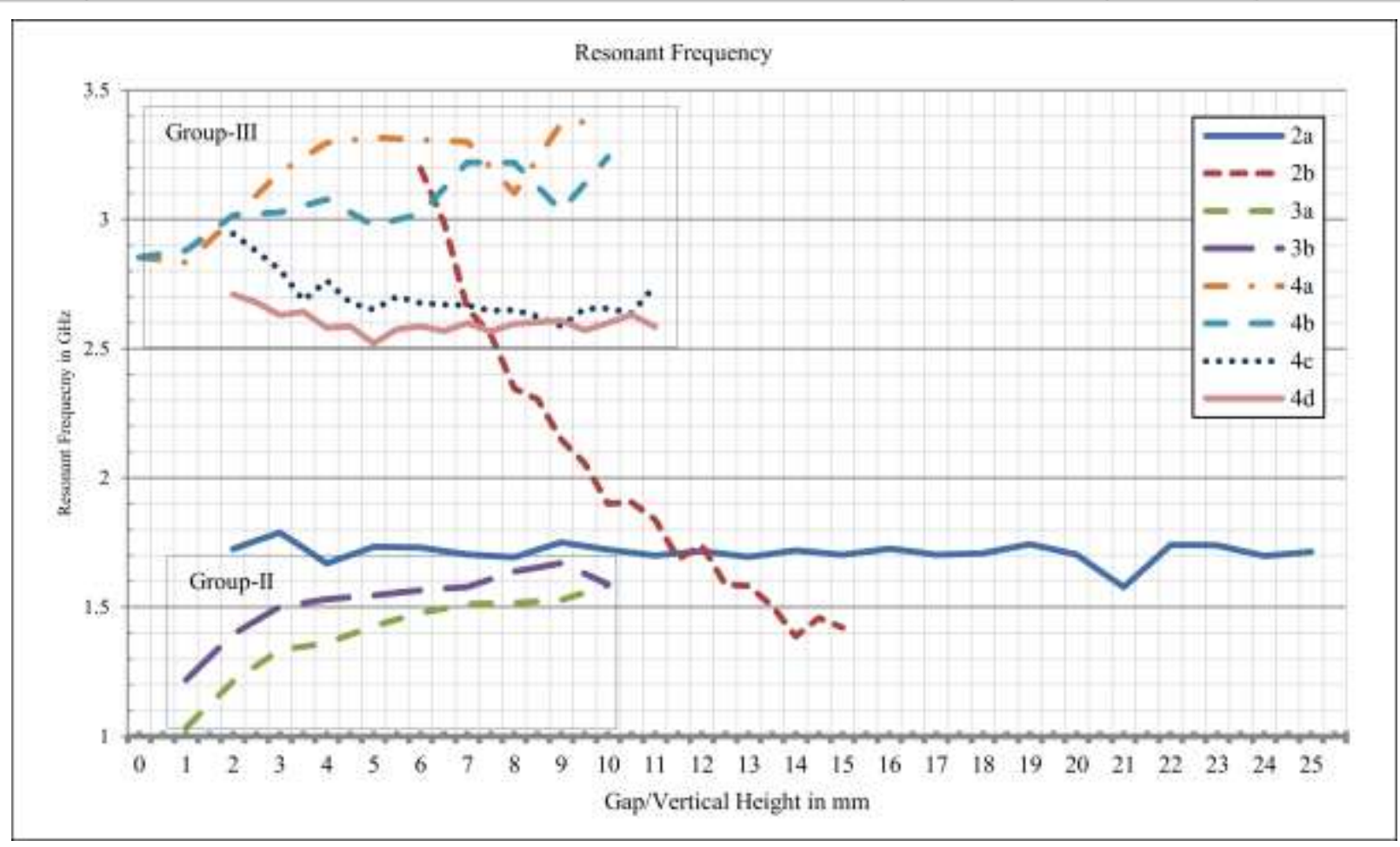

Figure 5 - Resonant frequencies for various configurations of SRs combined. 


\section{REFERENCES}

[1] C. Enkrich, M. Wegener, S. Linden, S. Burger, L. Zschiedrich, F. Schmidt, J. Zhou, T. Koschny and C. Soukoulis, "Magnetic Metamaterials at Telecommunication and Visible Frequencies", Physical Review Letters, vol. 95, no. 20, 2005.

[2] A., Castell, O., Barrow, D.A., Allender, C. and Porch, A. Masood, "Split ring resonator technique for compositional analysis of solvents in microcapillary systems," in The Proceedings of MicroTAS 2008 Conference, San Diego, 2008 October, pp. pp. 1636-1638.

[3] T., Ejaz, T., Naveed, M., Zaidi, T. and Rahman, H.U. Hayat, "Simulation Based Comparative Analysis of Split Resonators for Compositional Analysis.," in The Fourth International Conference on Technological Advances in Electrical, Electronics, 2016, September.

[4] H. Choi, J. Naylon, S. Luzio, J. Beutler, J. Birchall, C. Martin and A. Porch, "Design and In Vitro Interference Test of Microwave Noninvasive Blood Glucose Monitoring Sensor", IEEE Transactions on Microwave Theory and Techniques, vol. 63, no. 10, pp. 3016-3025, 2015.

[5] A. Abduljabar, D. Rowe, A. Porch and D. Barrow, "Novel Microwave Microfluidic Sensor Using a Microstrip Split-Ring Resonator", IEEE Transactions on Microwave Theory and Techniques, vol. 62, no. 3, pp. 679-688, 2014.

[6] O. Sydoruk, E. Tatartschuk, E. Shamonina and L. Solymar, "Analytical formulation for the resonant frequency of split rings", Journal of Applied Physics, vol. 105, no. 1, p. 014903, 2009.

[7] M. Mehdizadeh and T. Ishii, "Electromagnetic field analysis and calculation of the resonance characteristics of the loop-gap resonator", IEEE Transactions on Microwave Theory and Techniques, vol. 37, no. 7, pp. 1113-1118, 1989.

[8] W. Hardy and L. Whitehead, "Split-ring resonator for use in magnetic resonance from 200-2000 MHz", Review of Scientific Instruments, vol. 52, no. 2, pp. 213-216, 1981.

[9] T. Ejaz, Rahman, H.U., Shah, S.A.A., and Zaidi, T., "A comparative analysis of split-ring resonator models," in Proceedings of the 4th International Conference on Informatics, Electronics \& Vision (ICIEV'15), 2015, p. p. 61.

[10] D. Pozar, Microwave engineering, 1st ed. Hoboken, N.J.: Wiley, 2012

[11] C. K. Ong, C. P. Neo, V.V. Varadan and V.K. Varadan L.F.Chen, Microwave Electronics; Measurement and Material Characterization, 250286th ed.: John wily and sons.

[12] D. Rowe, S. al-Malki, A. Abduljabar, A. Porch, D. Barrow and C. Allender, "Improved Split-Ring Resonator for Microfluidic Sensing", IEEE Transactions on Microwave Theory and Techniques, vol. 62, no. 3, pp. 689-699, 2014.

[13] Xutao Tang, Iryna E. Khodasevych, and Wayne S. T. Rowe, "Tunable split ring resonators using air pressure," in Microwave Symposium (AMS), 2016 IEEE 2nd Australian, 2016.

[14] Ansoft HFSS software version 13. (2017, March) ANSYS HFSS. [Online]. http://www.ansys.com/products/electronics/ansys-hfss

[15] T. Ejaz, Shah, S.A.A., Rahman, H.U., and Zaidi, T., "Improved Shield Design for Split-Ring Resonator," in The Third International Conference on Technological Advances in Electrical, Electronics and Computer Engineering (TAEECE2015), Beirut, Lebanon, pp. Volume: ISBN: 9781-4799-5679-1.

[16] Hamood Ur Rahman, Syed Afaq Ali Shah, Tahir Zaidi Tahir Ejaz, "A Comparative Analysis of Split-Ring Resonator Models," in 4th International Conference on Informatics, Electronics \& Vision (ICIEV), Fukuoka, Japan, 2015 June.

[17] T. Ejaz, H. Ur Rahman, T. Tauqeer, A. Masood and T. Zaidi, "Shield Optimization and Formulation of Regression Equations for Split-Ring Resonator", Mathematical Problems in Engineering, vol. 2016, pp. 1-10, 2016.

\section{About Authors}

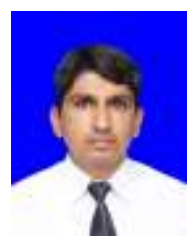

Muhammad Shoaib received the B.Sc degree in electrical engineering from College of Electrical and Mechanical Engineering, National University of Sciences and Technology, Islamabad, Pakistan. Presently he is working toward the Master degree in microwave engineering. His research concerns are design of tunable split ring resonators.

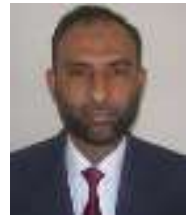

Muhammad Naveed received his Ph.D. degree from Quaid-i-Azam University, Islamabad, Pakistan in high frequency electromagnetics. Currently he is Associate Professor with Department of Electrical Engineering, College of Electrical and Mechanical Engineering, National University of Sciences and Technology, Islamabad, Pakistan.

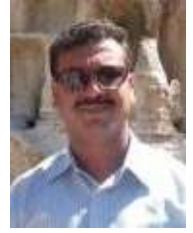

Tahir Ejaz received his B.Sc from University of Engineering and Technology, Lahore, Pakistan and M.Sc. degree from College of Electrical and Mechanical Engineering, National University of Sciences and Technology, Islamabad, Pakistan in electrical engineering. He is currently an Associate Professor with Department of Electrical Engineering, HITEC University, Taxila, Pakistan. His current research area is material characterization using microwave techniques.

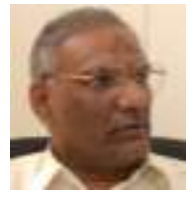

Tahir Zaidi received his Ph.D. degree from Georgia Institute of Technology - School of Electrical \& Computer Engineering, United States in 2010 in compound semiconductors. He is currently a Professor with Department of Electrical Engineering, College of Electrical and Mechanical Engineering, National University of Sciences and Technology, Islamabad.

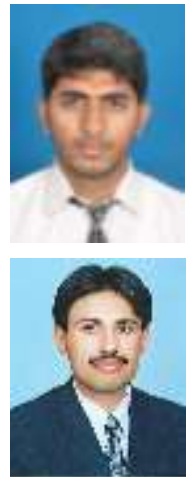

M. Haris Amir received the B.Sc degree in electrical engineering from Air University, Islamabad, Pakistan in 2014. Presently he is working toward the Master degree in Microwave Engineering at College of Electrical and Mechanical Engineering, National University of sciences and Technology, Islamabad, Pakistan. His current research area is microwave resonators.

Mashhood Ahmad received his Ph.D. degree from Quaid-i-Azam University, Islamabad in quantum optics. He worked on quantum state measurements schemes and entanglement dynamics. Currently he is at the faculty of Department of Electrical Engineering, College of Electrical and Mechanical Engineering, National University of Sciences and Technology, Islamabad. 